\title{
El Trabajo Interdisciplinar de Módulo como herramienta para el desarrollo de competencias transversales
}

\author{
Leire UGALDE \\ Elena BERNARAS \\ Eider RODRÍGUEZ \\ Ane ODRIA
}

Datos de contacto:

Leire Ugalde

Universidad del País Vasco

(UPV/EHU)

Departamento de Didáctica y

Organización Escolar

Elena Bernaras

Universidad del País Vasco

(UPV/EHU)

Departamento de Psicología Evolutiva y de la Educación

Eider Rodríguez

Universidad del País Vasco

(UPV/EHU)

Departamento de Didáctica de la Lengua y la Literatura

\section{Ane Odria}

Universidad del País Vasco (UPV/EHU)

Departamento de Didáctica de la Lengua y la Literatura

\section{RESUMEN}

El actual sistema universitario se enfrenta al reto de formar un alumnado capaz de adaptarse y dar respuesta a un contexto demográfico y socioeconómico en constante cambio. El desarrollo de competencias transversales se erige como clave para ello y los trabajos interdisciplinares de módulo pueden ofrecer el escenario propicio para su logro. Esta investigación presenta los resultados obtenidos en el desarrollo de seis competencias transversales a través de la aplicación del módulo "Diversidad en la Escuela" en el segundo curso del Grado de Educación Primaria de la Universidad del País Vasco (UPV/EHU). 113 estudiantes respondieron a un cuestionario, tanto antes como después de realizar el módulo, donde se les preguntaba acerca de su percepción sobre estas competencias. Además, se recogieron las respuestas a varias preguntas abiertas al final de la intervención. Los resultados mostraron que el estudiantado percibió una mejora significativa en su Capacidad Investigadora, el Razonamiento Crítico, el Tratamiento de la Información y la Transferencia del Conocimiento. El artículo concluye con las reflexiones sobre la aportación que estas mejoras conllevan en la formación de los futuros docentes.

PALABRAS CLAVE: Formación de docentes;
competencias;
interdisciplinariedad. 


\title{
The Modular Interdisciplinary Project as a tool for developing transversal competences
}

\begin{abstract}
The current university system faces the challenge of training students that will be able to adapt and respond to a constantly changing demographic and socioeconomic context. The development of transversal competencies by means of modular interdisciplinary projects provides an appropriate setting for achieving such goal. This research presents the results obtained in the training of six transversal competences through the module "Diversity at School" carried out in the second year of the Degree in Primary Education of the University of the Basque Country (UPV/EHU). 113 students answered a questionnaire concerning their perception with regards to such competences, both before and after the accomplishment of the module. Besides, the answers of certain open questions were also collected at the end of the intervention. The results showed that students perceived a significant improvement in their competence regarding research abilities, critical reasoning, treatment of information and transfer of the knowledge. The paper concludes by discussing the contribution that these improvements entail for the training of future teachers.
\end{abstract}

KEYWORDS: Teacher education; competences; learning packages; interdisciplinary approach.

\section{Introducción}

En los últimos tiempos la universidad se ha visto abocada a hacer frente a diversos retos. Por una parte, el proceso de creación de un Espacio Europeo de Educación Superior (EES, 2019a) ha implicado un cambio de paradigma en el que, pasando a un segundo plano el mero hecho de transmitir contenidos, se enseña a aprender, ofreciendo al alumnado nuevas herramientas para ello (Benito y Cruz, 2005; Ortega, 2010). De este modo, el alumnado podrá emprender su proceso de aprendizaje de forma activa, planteándose preguntas, investigando, seleccionando información, sacando conclusiones, etc. (Rekalde, Martinez y Marco, 2012). Por otra parte, la vertiginosa transformación social y productiva que se está dando en las últimas décadas, ha obligado a que la educación universitaria ajuste los procesos formativos al tiempo presente (Cowan, 2006). Hoy más que nunca es necesario formar al alumnado desde un punto de vista integral, enriqueciendo sus conocimientos, su capacidad técnica, personal y relacional (Delors, 1996). De este modo, las competencias genéricas y específicas de los planes de estudio de las diversas universidades europeas han pretendido definir las capacidades que ayudarán al alumnado a adaptarse con éxito a las demandas de la sociedad. El marco de referencia para definirlas fue establecido en el proyecto Tuning (EES, 2019b; González 
y Wagenaar, 2003).

Basándose en los principios de este marco europeo y con miras a adaptarse a este nuevo contexto, la Universidad del País Vasco (UPV/EHU) acometió la renovación de su Plan Estratégico de actuación en 2018 (UPV/EHU, 2018). Para ello, apostó por desarrollar un modelo educativo propio, el denominado IKD (Ikaskuntza Kooperatibo eta Dinamikoa/ Aprendizaje Cooperativo y Dinámico), incrementando el uso de metodologias innovadoras en el proceso de enseñanza-aprendizaje e impulsando el trabajo en equipos docentes estructurados. Este funcionamiento será la base para dar respuesta a una de las debilidades detectadas en el diagnóstico interno: la baja formación del alumnado en ciertas competencias transversales. De esta manera, se antoja necesaria la intensificación de algunas de ellas, como son, por ejemplo, el pensamiento crítico o la resolución de problemas (UPV/EHU, 2018).

Estos propósitos han de ser asumidos por los diversos centros y titulaciones que constituyen la UPV/EHU, siendo responsabilidad de los mismos realizar las adaptaciones curriculares necesarias que mejor se adecuen a los contextos propios de cada uno (Rekalde et. al., 2012). Con este fin, el diseño curricular del Grado de Educación Primaria de la Facultad de Educación, Filosofia y Antropología (FEFA) ha sido estructurado a través de módulos. La propuesta se concreta en cuatro módulos cuatrimestrales y un quinto anual que asegura la coordinación entre asignaturas del mismo curso (UPV/EHU, 2015). Estos permiten desarrollar las competencias propias de la titulación mediante la aplicación de metodologías activas que favorecen la interconexión de las diversas materias en un Trabajo Interdisciplinar de Módulo (TIM). Este tipo de diseño responde a los retos planteados por la UPV/EHU, ya que favorece un modelo docente más práctico, activo y colaborativo (Rekalde et al., 2012), y contribuye al desarrollo de las competencias transversales (Aristizabal, Rodríguez-Fernández, Rodríguez-Miñanbres y FernándezZabala, 2015; Rodríguez-Miñambres, Rico-Martínez, López de SosoagaLópez de Robles y Ugalde-Gorostiza, 2018). Sin embargo, es necesario seguir ahondando en la recopilación de evidencias que muestren la relación existente entre los TIM y el desarrollo de competencias.

Con el fin de ayudar en esta tarea, el presente trabajo muestra los resultados obtenidos a través de la implementación del módulo denominado "Diversidad en la Escuela" en relación al desarrollo de seis competencias transversales: Capacidad Investigadora, Tratamiento de la Información, Transferencia del Conocimiento, Razonamiento Crítico, Gestión de Conflictos y Trabajo en Equipo.

\section{El módulo "Diversidad en la Escuela" como herramienta para el desarrollo de competencias transversales}


El módulo "Diversidad en la Escuela" se lleva a cabo en el segundo cuatrimestre del segundo curso del Grado de Educación Primaria, constituyéndose como el cuarto módulo que el alumnado ha de realizar para completar el Grado. Conformado por cuatro asignaturas (Organización escolar, Didáctica de la lengua en contextos multilingües, Bases de la escuela inclusiva y Dificultades en el desarrollo y el aprendizaje), tiene como objetivo primordial capacitar al alumnado para responder a un sistema educativo inclusivo, en el que la escuela ofrezca una educación de calidad, independientemente de las características sociales, personales y culturales del alumnado (UNESC, 1994). El futuro profesorado ha de asumir que la educación debe ser para todos (UNESCO, 1990; 2015; 2016) y las escuelas han de desarrollar culturas, politicas y prácticas inclusivas que ayuden a superar cualquier barrera de participación o aprendizaje (Booth y Aiscow, 2002; Gobierno Vasco, 2019). Para ello, es necesario que el profesorado adquiera las competencias necesarias para atender la diversidad con éxito.

Las competencias básicas, también denominadas genéricas o transversales (Aristizabal et al., 2015; Rodríguez, Ibarra y Cubero, 2018), son clave para capacitar al profesorado. Como indican Flecha, Racionero, Tintoré y Arbós (2014) refiriéndose a las recomendaciones recogidas en el informe del 2012 Rethinking Education de la Comisión Europea, es importante trabajar las competencias transversales para desarrollar la capacidad de pensar críticamente, de resolver problemas o trabajar en colaboración, entre otras. Estos autores subrayan la importancia de que la formación inicial ha de ejercitar al futuro cuerpo docente en el desarrollo de habilidades de investigación y reflexión, y argumentan que los países que tienen mejores resultados en su educación primaria y secundaria basan la formación del profesorado en las mejores evidencias científicas del área. Muy al contrario, actualmente existen evidencias de que un gran número de docentes tiende a priorizar el conocimiento adquirido a través de su propia experiencia o de los consejos recibidos a través de colegas frente al derivado de la investigación científica educativa, recelosos de que esta última pueda realizar algún aporte significativo en la práctica en el aula (Diaz Costa, Fernández Cano, Faouzi y Caamaño, 2019). Este tipo de consideraciones facilitan la expansión de creencias erradas, como podrían ser los neuromitos (Dekker, Lee, Howard-Jones y Jelle, 2012; HowardJones, 2014; Karakus, Howard-Jones y Jay, 2015), que se consolidan e influyen negativamente en el futuro profesorado (Fuentes y Riso, 2015).

Es de suma importancia, por lo tanto, formar en un conocimiento sólido y con bases científicas, derribando lo que Perines (2017) ha denominado como las "murallas invisibles" que existen entre la investigación y la práctica docente. Para ello será necesario que el futuro profesorado sea capaz de transferir y aplicar lo aprendido (Gairin, 2011). Las estrategias para alcanzar este fin pueden ser varias como, por ejemplo, el Estudio de 
Casos o el Aprendizaje Basado en Problemas (Gairín, 2011; Moreno, 2012). A través de estas metodologías el profesorado deberá proporcionar a los estudiantes situaciones de aprendizaje contextualizadas, cercanas a problemáticas reales, de manera que estos desarrollen su capacidad para hacer uso pertinente del contenido disciplinar en la resolución de problemas (Fernández, 2006).

Frente a todos estos retos, el módulo "Diversidad en la escuela" propone un TIM que fue creado en 2012, habiendo sido evaluado y reelaborado año tras año, y finalmente consolidado como Proyecto de Innovación Educativa (PIE) para el bienio 2018-2019 (Convocatoria PIE 2017-2018 del Servicio de Asesoramiento Educativo de la UPV/EHU). Este TIM pretende desarrollar tanto las competencias especificas de cada asignatura como las competencias transversales. En él se presentan 8 casos que escenifican situaciones de diversidad en el aula, validados por un grupo de asesores del Servicio de Apoyo e Innovación (Berritzegune) del Gobierno Vasco y basados en las peticiones más habituales que en el último año académico han recibido de los centros educativos. Los temas presentados fueron los siguientes: 1. problemática lingüistica en el entorno escolar; 2 . discapacidad intelectual; 3. altas capacidades; 4. respuesta educativa al alumnado inmigrante; 5. adopción; 6. problemas emocionales ligados a baja autoestima; 7. violencia de género; 8. transexualidad. Estos casos se redactan en base a las competencias a desarrollar en cada asignatura, con el fin de que el alumnado pueda responder a cada uno de ellos de manera global e integral y desde un punto de vista interdisciplinar.

\section{Descripción y desarrollo del TIM “Diversidad en la Escuela"}

El alumnado, formado en grupos de 4 o 5 personas, elige uno de los casos entre los ocho que se les plantean. El reparto de los casos está guiado por la norma de que todos los casos han de quedar repartidos entre los grupos existentes. Una vez asignado el caso, cada grupo ha de llegar a un acuerdo de trabajo para asegurar el correcto funcionamiento del mismo. Este acuerdo se revisa y reelabora durante el proceso, incorporando los cambios que puedan ayudarles a garantizar una óptima colaboración en el grupo. A través de rúbricas se les ayuda a conformar el marco que asegure y garantice que los principios consensuados se respeten en todo momento y a lo largo de todo el proceso. Se entrega al alumnado una rúbrica modelo basada en la propuesta realizada por Chica Moreno (2011) y se les explica cómo realizar la evaluación de grupo, la coevaluación y la evaluación individual siguiendo las propuestas de Johnson \& Johnson (2014). Estas rúbricas y pautas, proporcionan tanto al alumnado como al profesorado una herramienta útil para regular, gestionar y evaluar el proceso de trabajo realizado por cada uno de los miembros del grupo.

Una vez realizados los grupos y distribuidos los casos, cada grupo 
analiza las claves del problema, consulta la bibliografia pertinente, elabora un marco teórico, define los objetivos de intervención, diseña una intervención basada en evidencias científicas y redacta las conclusiones. El trabajo final ha de presentarse de dos formas: a) por un lado, un artículo académico (con un abstract en euskera e inglés, palabras clave, marco teórico, objetivos, intervención, conclusiones y referencias) redactado según las normas establecidas por la American Psychological Association (APA, 2010); b) por otro, una presentación oral de 10 minutos con posterior debate, en el que participará toda la clase. La presentación sigue el mismo formato de las comunicaciones orales en los congresos. Ayudado por el soporte que escojan (power point, prezi etc.), cada grupo presenta una sintesis del trabajo elaborado. Una vez finalizada la presentación, se abre el turno de preguntas que han de realizar quienes han escuchado la presentación. Un tribunal compuesto por dos docentes modera, guía y evalúa todo el proceso y el contenido presentado.

La metodología propuesta para elaborar el trabajo se centra en el Aprendizaje Basado en Problemas, ya que permite desarrollar la capacidad investigadora, el trabajo colaborativo o el pensamiento crítico, siempre y cuando se planifique e incluya como meta el aprendizaje de este último (Morales, 2018). Durante el proceso se realiza un constante seguimiento y tutorización del trabajo por parte del profesorado. El profesorado que compone el modulo se distribuye en parejas multidisciplinares (de distintas asignaturas y departamentos) para proporcionar el feedback a los dos informes que el alumnado entrega durante el proceso, así como para realizar la evaluación del trabajo y presentación final. El primer informe reúne una breve descripción del caso junto con la identificación de las principales claves que destacan en él, las cuales servirán para definir los objetivos provisionales de la intervención. En el segundo, el alumnado redacta el marco teórico que sustentará la intervención, y partiendo de este marco, se establecen los objetivos definitivos. La tutorización directa se complementa con otras actividades, como por ejemplo: talleres sobre la utilización de bases de datos científicas, uso de normas APA, charlas y seminarios de los temas a tratar, presentación y análisis de modelos de trabajos presentados en cursos anteriores, etc.

Así, el módulo "Diversidad en la Escuela" pretende desarrollar 6 competencias transversales (ver tabla 1): 


\section{Tabla 1}

Competencias transversales planteadas en el módulo

Código

Competencia

C1. Capacidad
Investigadora

C1. Capacidad
Investigadora

Desarrollar la capacidad investigadora: ser capaz de buscar la información en bases de datos científicas y elaborar un marco teórico sobre el que sustentar las decisiones que se tomen como profesional.

C2. Tratamiento de la Información
Distinguir la información que cuenta con validez científica, utilizar como criterio de discriminación la fuente de información, identificar la información clave, relacionar ideas y redactarlas en formato académico siguiendo las pautas de redacción establecidas en el APA $6^{\circ}$ edición.

Transferir el conocimiento derivado de la revisión de documentación científica y del conocimiento adquirido en las diversas disciplinas a las situaciones prácticas planteadas de forma efectiva.
C3. Transferencia del Conocimiento
Reflexionar sobre la práctica educativa de forma critica, tomar decisiones sobre la misma infiriendo la resolución más adecuada de la información derivada de las investigaciones científicas.

C5. Gestión de Conflictos

Gestionar los conflictos de forma positiva, convirtiéndolos en una posibilidad para mejorar el funcionamiento grupal, a través de la reflexión, evaluación y reelaboración de los acuerdos y procesos de trabajo.

C6. Trabajo en Equipo

Trabajar en equipo de forma efectiva (incentivando la interacción, creando aprendizaje de forma colaborativa, reflexionando conjuntamente, respetando los argumentos de validez, realizando una escucha activa, etc.)

\section{Propósito de la investigación}

Este trabajo responde a un doble propósito: por un lado, surge del 
interés del equipo docente de evaluar en qué medida el TIM cumple con los requisitos necesarios para capacitar al alumnado en algunas de las competencias básicas necesarias para desarrollar una buena labor profesional en el futuro; por otro lado, pretende aportar información que ayude a dar respuesta a la falta de literatura detectada de cómo y en qué medida se trabajan las competencias transversales (Eizagirre, Altuna, Pikabea, Marko y Perez, 2017).

Siguiendo el planteamiento defendido por Rodríguez et al. (2018) en el Proyecto de excelencia Re-Evalua, sabemos que el alumnado es capaz de realizar un juicio acertado de sus competencias y que esta percepción influye en el aprendizaje y desarrollo de las mismas. En el marco teórico sobre el que se sustenta el proyecto, Rodríguez et al. (2018) señalan dos aspectos clave referidos al desarrollo de competencias básicas. Por una parte, refieren investigaciones que muestran cómo la participación del alumnado en el proceso de evaluación (autoevaluación, evaluación entre iguales y heteroevaluación, procesos que se desarrollan en el TIM "Diversidad en la Escuela") ayuda a mejorar el desarrollo de competencias. Y por otro lado, recaban resultados de estudios en los que se demuestra la importancia de la autoeficacia percibida por el estudiantado y su relación directa con el desarrollo de competencias, siendo esta percepción de la autoeficacia un criterio a tener en cuenta como predictor del éxito del estudiante.

Tomando como base estos principios, en este estudio se plantearon dos objetivos en torno a las seis competencias transversales que se trabajan en el módulo:

1. Identificar la percepción que tienen los estudiantes al inicio del módulo sobre las seis competencias transversales.

2. Determinar si existen diferencias en la percepción del desarrollo de competencias transversales tras la implementación del TIM.

\section{Método}

\section{Participantes}

La muestra estuvo compuesta por 113 estudiantes que cursaron el segundo curso del Grado de Educación Primaria en el curso escolar 20182019 en el Campus de Gipuzkoa de la Universidad del País Vasco (UPV/EHU). Su edad oscilaba entre los 18 y 27 años $(M=19,65 ; S D=1,27)$. El $69 \%$ se autodefinió como chica $(\mathrm{n}=78)$, el $30,1 \%$ como chico $(\mathrm{n}=34)$ y el $0,9 \%$ se identificó en la categoría de no binario $(\mathrm{n}=1)$.

\section{Instrumento y procedimiento}

Antes de dar comienzo al TIM, la primera semana de clase del segundo 
cuatrimestre (finales de enero), se explicó al alumnado el objetivo de la investigación. Con objeto de respetar en todo momento la confidencialidad y el anonimato, se les indicaron las pautas a seguir para suplantar su identidad por un código (compuesto por la primera letra de su nombre y los cuatro últimos dígitos del número del carnet de identidad).

El alumnado cumplimentó en un tiempo aproximado de 20-25 minutos de forma digital, en el propio aula y en presencia de una persona del equipo docente un cuestionario diseñado ad-hoc. El cuestionario estuvo compuesto por un total de 58 ítems, con formato de respuesta tipo Likert en una escala ascendente de cinco puntos. Los cinco primeros ítems del cuestionario fueron diseñados para recoger datos identificativos de las personas encuestadas (como edad, sexo y estudios previos realizados). Los 53 items restantes, cumplian con la finalidad de medir la percepción del alumnado sobre las seis competencias objeto de estudio.

Para ello, se utilizaron preguntas en las que, en ocasiones, el alumnado debía mostrar acuerdo o desacuerdo (1: Nunca, 5: Siempre) con una afirmación (item 58: Eres capaz de basar tus decisiones en evidencias científicas a la hora de dar respuesta a tu práctica educativa en el aula), señalar la frecuencia (1: Nunca, 5: Siempre) con la que realiza una acción concreta (item 36: ¿Has utilizado alguna vez las normas APA?, o ítem 40: ¿Has realizado alguna vez búsquedas en bases de datos como: WOS, ERIC, SCOPUS o Sciencedirect?) o cuantificar el nivel de validez (1: Ninguna, 5: Total) de diversas fuentes (Señala cuál es la validez científica de las siguientes fuentes, item 44: un blog, ítem 45: la noticia de un periódico, item 46: una revista divulgativa, item 47: una revista cientifica, etc.). Además, se incluyó el Cuestionario de Aprendizaje Cooperativo de Fernández-Río, Cecchini, Méndez-Giménez, Méndez-Alonso y Prieto (2017), para medir la habilidad de aprender y trabajar de forma cooperativa dentro de la competencia de Trabajo en Equipo.

Al finalizar el cuatrimestre, se repitió el mismo procedimiento, invitando al alumnado a completar de nuevo ambos cuestionarios.

Por último, cada grupo de trabajo (34 grupos en total) entregó, junto con el trabajo final, la respuesta a cinco preguntas abiertas en las que se les pedía su valoración sobre el módulo. Estas preguntas se referían a cuatro áreas: 1) obtención de las competencias previstas, 2) valoración del proceso y material de trabajo, 3) valoración de la implicación del profesorado y del alumnado y 4) sugerencias para mantener o cambiar la propuesta de trabajo y justificación de su respuesta.

\section{Análisis de datos}

Los análisis estadísticos de los datos recogidos a través de los cuestionarios se realizaron a través del paquete informático IBM SPSS $®$ versión 20 para Windows, con el que se llevaron a cabo análisis estadísticos descriptivos y de frecuencias, y la prueba no paramétrica $\mathrm{T}$ de Wilcoxon para el análisis de diferencias intrasujeto para muestras relacionadas, por 
no cumplirse el supuesto de normalidad.

Las respuestas a las preguntas abiertas fueron analizadas en una reunión con todo el profesorado del TIM (9 personas en total), en la que se analizaron los logros obtenidos y se concretaron futuras propuestas de mejora.

\section{Resultados}

\section{Percepción de las competencias antes de la implementación del TIM (Pre-test)}

Antes de la realización del TIM, la competencia peor valorada por el alumnado fue la Capacidad Investigadora $\left(M_{\text {pre }}=2,36\right)$. En un nivel intermedio se situaron la Gestión de Conflictos $\left(M_{\text {pre }}=3,38\right)$, el Razonamiento Crítico $\left(M_{\text {pre }}=3,46\right)$, el Tratamiento de la Información $\left(M_{\text {pre }}=\right.$ $3,53)$ y la Transferencia del Conocimiento $\left(M_{p r e}=3,53\right)$ y la mejor valorada fue la competencia de Trabajo en Equipo $\left(M_{\text {pre }}=4,03\right)$.

\section{Comparación de la percepción de las competencias después de implementar el TIM (Post-test)}

Una vez realizado el TIM, las cuatro competencias mejor evaluadas y que presentaron diferencias significativas fueron las siguientes (ver figura 1): Razonamiento Crítico $\left(M_{\text {pre }}=3,46\right.$ y $\left.M_{\text {post }}=3,99 ; \mathrm{z}=6,67, \mathrm{p}<0,01\right)$, Capacidad Investigadora $\left(M_{\text {pre }}=2,36\right.$ y $\left.M_{\text {post }}=2,84 ; \mathrm{z}=7,67, \mathrm{p}<0,01\right)$, Tratamiento de la Información $\left(M_{\text {pre }}=3,52\right.$ y $\left.M_{\text {post }}=3,99 ; \mathrm{z}=7,26, \mathrm{p}<0,01\right)$ y Transferencia del Conocimiento $\left(M_{\text {pre }}=3,53\right.$ y $M_{\text {post }}=3,96 ; \mathrm{z}=7,15, \mathrm{p}<$ $0,01)$. Los tamaños del efecto de todas las diferencias fueron altas $(\mathrm{r}=0,62-$ $0,72)$.

No se encontraron diferencias estadísticamente significativas en las competencias de Trabajo en Equipo y Gestión de Conflictos ( $p>0,05)$.

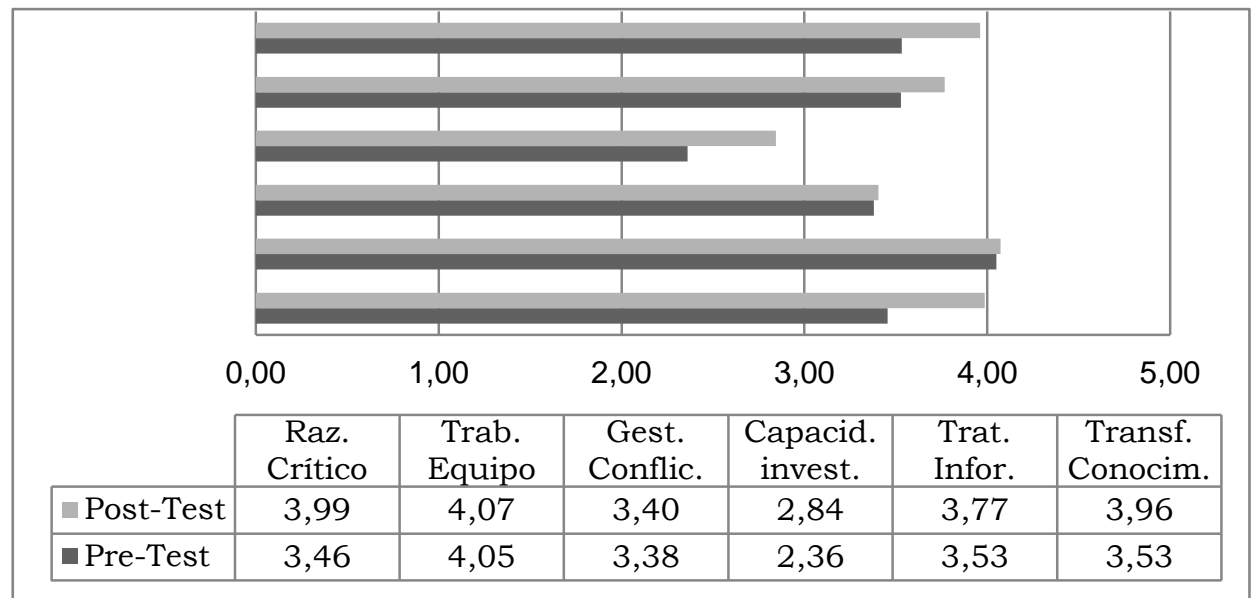

Figura 1. Percepción del alumnado de su nivel de competencias antes y después de la implementación del TIM. 


\section{Mejoras percibidas en cada competencia}

\section{Capacidad Investigadora}

El alumnado inicia el cuatrimestre con una percepción negativa respecto a su Capacidad Investigadora, siendo esta la competencia peor valorada $\left(M_{\text {pre }}=2,36\right)$. Se ha podido constatar una muy baja tendencia a utilizar bases de datos especializadas. Solo un 15,1\% del alumnado dice utilizar estas bases de datos alguna vez, frente a un 91,2\% que afirma utilizar, siempre o casi siempre, Internet en general. Esta tendencia mejora con la participación en TIM que logra que el 40,7\% del alumnado utilice, a menudo o siempre, bases de datos especializadas para realizar sus trabajos académicos.

\section{Tratamiento de la Información}

Los resultados mostraron que existía una gran confusión inicial entre los participantes sobre la validez que debian otorgar a la información derivada de diversas fuentes. En la figura 2 se observa que los resultados del pre-test indicaban que la información procedente de las revistas divulgativas contaba con una gran o total fiabilidad para el 54\% del alumnado, seguida de los apuntes de un compañero $(52,2 \%)$. También las noticias publicadas en los periódicos eran valoradas con un alto o completo nivel de fiabilidad por el $45 \%$ de la muestra. Sin embargo, solo un $42 \%$ de los participantes otorgaba ese mismo nivel de confianza a las revistas científicas o a los trabajos Fin de máster (38\%) que se posicionaban en los niveles más bajos, a la par de los apuntes de asignaturas $(37,2 \%)$ o la información aportada en un blog (33\%).

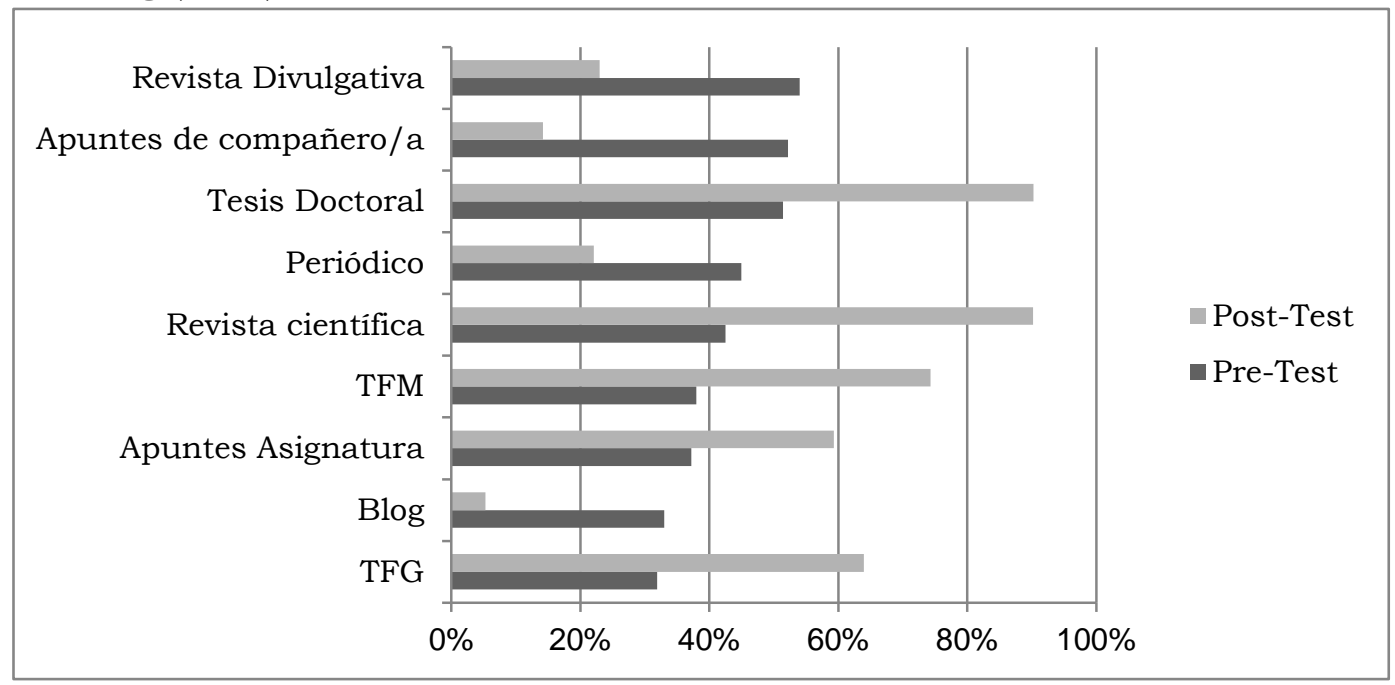

Figura 2. Comparación de porcentajes de alumnado que otorga alta o total fiabilidad a la información derivada de diversas fuentes.

Los resultados post-test, sin embargo, señalan una gran subida en la 
confianza que el alumnado deposita en la información publicada en revistas científicas $(90,2 \%)$ y tesis doctorales $(90,3 \%)$, pasando a ser esta la fuente a la que otorgan mayor validez. Al contrario, dejan de considerar las revistas divulgativas (23\%), los apuntes de compañeros/as (14,2\%) o las noticias de periódicos $(22,1 \%)$ como información de alta relevancia científica.

Estos datos son coherentes con la importancia que el alumnado otorgaba al inicio del cuatrimestre a la procedencia de la información, siendo solo un $33,6 \%$ del alumnado quien, a menudo o siempre, tenía en cuenta que la información que manejaba procediera de bases de datos científicas. Este dato mejora con los datos post-test, donde un 64,6\% indica dar gran importancia a que la información proceda de estas bases de datos y un $72 \%$ dice utilizar como criterio de discriminación la fuente (frente a un 54,8\% del pre-test).

En cuanto al Tratamiento de la Información, la percepción de su capacidad para identificar el contenido más significativo, relacionar ideas y construir un marco teórico también mejora al final del cuatrimestre (Pre $68,1 \%$ - Post $87,6 \%)$.

Por último, a la hora de presentar la información de forma escrita, al inicio del cuatrimestre solo un 28,3\% afirmaba saber hacerlo respetando las normas APA. Este porcentaje incrementa hasta un 74,4\% al finalizar el TIM.

\section{Transferencia del Conocimiento}

Aunque el alumnado valora moderadamente bien su capacidad de transferir el conocimiento teórico a un caso práctico $\left(M_{p r e}=3,53\right)$, más de la mitad (55,8\%) afirma tener dudas sobre los pasos a seguir a la hora de responder a un problema educativo, y cerca de la mitad $(46,9 \%)$ tendría dificultades para definir los objetivos en el diseño de intervención. Estos aspectos mejoran considerablemente al final del cuatrimestre, donde los resultados muestran que un $70,8 \%$ del alumnado encuestado se muestra bastante o totalmente seguro de conocer los pasos que deberían seguir para responder a diversos retos educativos. Un $83,2 \%$ sabría definir los objetivos de intervención, un $85,9 \%$ se siente capaz de identificar las claves más importantes del problema a resolver y el $86,7 \%$ (frente a un 66,3\% inicial) considera que es capaz de transferir el conocimiento teórico a un caso práctico.

\section{Razonamiento Crítico}

El cambio más significativo en este ámbito se dio en la transformación que el alumnado percibió después de la intervención en su capacidad para identificar $\mathrm{y}$ basarse en evidencias cientificas y poder utilizar esta información de forma crítica en situaciones concretas relacionadas con su práctica profesional. De este modo, de un 49,6\% de las personas encuestadas que, inicialmente, señalaban tener grandes dudas respecto a su capacidad para utilizar de forma apropiada las evidencias científicas, 
solo un $16,8 \%$ se considera moderadamente capacitada al final de la intervención, frente a un $83,2 \%$ que siente bastante o plena confianza para hacerlo.

\section{Trabajo en Equipo y Gestión de Conflictos}

No se han observado mejoras significativas en las puntuaciones pre y post intervención en las competencias de Trabajo en Equipo ni en la Gestión de Conflictos, pero existen algunos aspectos que seria interesante tener en cuenta.

Así, prácticamente la totalidad de los y las estudiantes $(95,6 \%)$ ha trabajado en grupo con mucha frecuencia, pero no tiene costumbre de crear acuerdos de regulación de normas, ya que solo un 25,7\% dice realizar estos acuerdos con regularidad, y no tiene el hábito de levantar acta de las reuniones $(65,5 \%$ no lo hace nunca o casi nunca). Aunque el trabajo modular les ha ofrecido herramientas para poder realizar acuerdos $y$ levantar actas, no se ha observado una mejora relevante en estos aspectos (27,7\% realiza acuerdos siempre y un 55,7\% sigue sin levantar actas).

Por el contrario, obtienen muy buenas puntuaciones, tanto en el pre como en el post-test, en las cinco dimensiones que miden el trabajo cooperativo, oscilando todas las puntuaciones entre $M=4,21$ y $M=4,41$.

En cuanto a la Gestión de Conflictos se refiere, los resultados muestran que el $61,9 \%$ apenas ha tenido conflictos en el Trabajo en Equipo y un $59,3 \%$ a menudo o siempre ha sido capaz de solucionarlos. La causa que mayormente genera estos conflictos $(71,7 \%)$ es el trabajo desigual que realizan los participantes del grupo.

\section{Resultados cualitativos después de implementar el TIM}

En la información cualitativa recogida a través de las preguntas abiertas se observó un amplio consenso al afirmar que, a través del trabajo TIM, han conseguido adquirir las competencias propuestas. La clave para ello estaría en las características del trabajo planteado: "Creemos que este tipo de trabajo ofrece muy buenas posibilidades para trabajar estas competencias y, además, hemos tenido la posibilidad de ir dándonos cuenta de cómo las íbamos adquiriendo" (grupo 2). El TIM, además, les ha ayudado a generar un pensamiento crítico: "Este proceso nos ha llevado a realizar una reflexión crítica, y a generar un pensamiento crítico" (grupo 14) y a aprender a redactar un texto científico construido como resultado del trabajo en grupo: "Creemos que hemos adquirido las competencias. Hemos dado respuesta al caso que se nos planteó y para ello hemos tenido que leer diversas investigaciones para, a continuación, crear un marco teórico. De esta forma, hemos aprendido a escribir un texto científico y además, se ha incentivado el trabajo en grupo" (grupo 20).

Muchos de los grupos definen el trabajo propuesto como exigente, intenso y de una dificultad académica superior a la que están acostumbrados: "Este trabajo modular nos ha exigido una mayor implicación, ya que al 
plantearnos situaciones reales y tener que diseñar la solución más apropiada, hemos tenido que trabajar con mayor seriedad y rigor. Aun así, no planteariamos otro tipo de trabajo, el tema nos ha gustado mucho" (grupo 5) o: "Aunque sea un trabajo duro, hemos estado a gusto. Comparándolo con los trabajos modulares que hemos hecho hasta ahora, este nos ha parecido más complejo" (grupo 23).

Para la mayoría, es la primera vez que se enfrentan a la redacción de un artículo académico de estas características y resulta un reto importante. Sin embargo, lo valoran positivamente, y prácticamente el total de los grupos apuesta por mantener este módulo en el futuro sin realizar cambios significativos en el mismo: "No planteariamos otro tipo de trabajo porque consideramos que ha sido un trabajo interesante y con el que se aprende mucho" (grupo 3) o "No cambiariamos nada. Se nos han planteado temas interesantes y actuales, de modo que, mantendríamos esta misma propuesta para el alumnado del curso que viene" (grupo 29).

Destacan, no solo el aprendizaje derivado de cómo elaborar un marco teórico basándose en evidencias científicas, sino también la posibilidad que se les ofrece para reflexionar de manera crítica acerca de las intervenciones educativas: "Hemos podido ejercitarnos en la habilidad de identificar la diversidad en el aula, hemos aprendido a buscar fuentes con validez científica, hemos reflexionado sobre la situación desde una perspectiva crítica..." (grupo 31) y de conocer las piezas necesarias para elaborar un artículo académico: "No cambiaríamos nada. Creemos que es muy valioso aprender a redactar textos científicos del ámbito educativo, así como aprender a evaluar desde una perspectiva crítica las fuentes de información que utilizaremos en nuestro futuro como profesionales" (grupo 8).

Uno de los aspectos mejor valorados ha sido la diversidad de situaciones planteadas y, sobre todo, la relación directa de estas con la realidad. Esta última idea ha sido una de las más mencionadas. Consideran que estas situaciones pueden darse en el día a día de cualquier escuela y que es muy posible que en su futuro profesional deban gestionarlas: "Nos parece un trabajo apropiado, ya que son situaciones que podemos encontrar en el día a dia $y$, al fin $y$ al cabo, gracias a esto podemos ampliar nuestro conocimiento. Esto nos parece indispensable para nuestro futuro profesional" (grupo 9). Es por eso por lo que consideran indispensable y de gran utilidad para su futuro el aprender a formular respuestas adecuadas y basadas en evidencias cientificas: "Nos parece un trabajo modular muy útil por la relación tan estrecha que tiene con la realidad, ya que es muy probable que en un futuro nos toque diseñar propuestas como esta" (grupo 26). Creemos necesario señalar que este vínculo percibido entre la realidad y las situaciones planteadas ha sido un factor motivador a la hora de abordar el trabajo: "Nos hemos sentido muy motivados porque el tema nos ha parecido muy interesante desde el principio. Además, creemos que este trabajo será de gran utilidad para nuestro futuro profesional porque nos puede tocar gestionar una situación parecida en el aula" (grupo 33). 


\section{Conclusiones}

La universidad en general, y la UPV/EHU en particular, ha apostado claramente por trabajar en pro de una educación de calidad que capacite al alumnado para dar respuesta a una sociedad en continuo cambio (UPV/EHU, 2018). Las competencias transversales se erigen como una de las claves para ello, por consiguiente, es necesario que la planificación curricular de cada centro se adapte (Rekalde et al., 2012) para asegurar su desarrollo. Estructurar la titulación a partir de módulos es la propuesta que desde la Facultad de Educación, Filosofia y Antropología (FEFA) de la Universidad del País Vasco se ha realizado para la titulación de Grado de Primaria. Estos módulos favorecen un modelo docente más práctico, activo y colaborativo (Rekalde et al., 2012), impulsando el trabajo en equipos docentes coordinados (UPV/EHU 2018) y diluyendo las barreras existentes entre distintas disciplinas (Rodríguez et al., 2018). Sin embargo, resulta necesario evaluar cómo y en qué medida los módulos ayudan a desarrollar las competencias transversales (Eizagirre et al., 2017) para aportar evidencia de su eficacia. Los resultados obtenidos en esta investigación ayudan a confirmar que el TIM denominado "Diversidad en la escuela" es una propuesta valida para trabajar las competencias transversales, competencias que se consideran importantes para mejorar las capacidades profesionales del futuro profesorado.

De las seis competencias definidas, en cuatro (Capacidad Investigadora, Razonamiento Crítico, Tratamiento de la Información y Transferencia del Conocimiento) el alumnado ha percibido una mejora significativa tras la implementación del TIM. Gracias a este, el alumnado dice sentirse más capacitado para basar sus decisiones en evidencias científicas y utilizar la información obtenida de forma crítica en situaciones prácticas. Acercar al alumnado a las bases científicas y fundamentar su formación en base a las mismas es una de las claves para mejorar la formación del futuro profesorado, alejando del aula las prácticas basadas en creencias erróneas y generando intervenciones efectivas. Para ello, es necesario que el profesorado rompa con la nefasta opinión que tiene del impacto que la investigación puede tener en el aula (Costa et al., 2019) y le ofrezca mayor credibilidad. Con los resultados de este estudio se constata que este módulo ha conseguido una mejora en este cambio de percepción, ya que ha incrementado significativamente el grado de validez que el alumnado otorga a las revistas científicas, así como a la procedencia de la información en la elaboración de un trabajo académico. Además, el alumnado percibe una mejora competencial en la Transferencia del Conocimiento a situaciones prácticas, capacidad indispensable para una buena capacitación docente (Gairin, 2011).

La falta de mejoras significativas detectada en las competencias de Trabajo en Equipo queda justificada por el alto nivel del que partía esta 
competencia desde el inicio. El alumnado está habituado a trabajar en equipo y considera que tiene la capacidad suficiente para ello. Los resultados obtenidos, tras analizar todas las dimensiones del trabajo cooperativo, asi lo demuestran.

No obstante, la falta de mejora en la Gestión de Conflictos merece una reflexión. Los conflictos que surgen en los equipos de trabajo suelen ser una fuente de preocupación reiterada para los docentes. Para hacer frente a este problema se han realizado acuerdos que se han reelaborado durante el proceso dependiendo de los conflictos surgidos, pero estos intentos no han logrado una mejora significativa en esta competencia. Será necesario incorporar cambios en el módulo que ayuden a mejorar este aspecto, realizando por parte del profesorado un seguimiento más exhaustivo de la aplicación que el alumnado hace de estas técnicas.

Por último, es preciso señalar, que las opiniones del alumnado recogidas mediante preguntas abiertas han corroborado los resultados obtenidos a través del cuestionario. El alumnado valora positivamente el trabajo realizado en el módulo y considera que es válido para desarrollar las competencias transversales propuestas, y aunque consideran que el trabajo planteado es exigente y con un nivel de dificultad alto, se sienten motivados para realizarlo. Plantear situaciones de análisis contextualizadas y cercanas a problemáticas reales (Fernández, 2006) ha contribuido a generar esta motivación. Asimismo, la metodología utilizada, que les ha posibilitado trabajar de forma activa, planteándose preguntas, investigando, seleccionando información, sacando conclusiones... (Rekalde et al., 2012) ha sido de gran ayuda. Aun asi no podemos olvidar que, durante todo el proceso, ha existido un continuo seguimiento por parte del profesorado que a través de diversas actividades ha asegurado que se ofrecian las herramientas necesarias para la correcta elaboración del trabajo, planificando y garantizando que se llevasen a cabo los procesos necesarios para generar un pensamiento crítico (Morales, 2018). Esta sensación de ser capaces de enfrentarse con éxito a una tarea, definida por ellos mismos como exigente y dificil, ha sido otra de las fuentes generadoras de motivación. Al final del módulo los y las estudiantes han percibido una mejora en su capacidad para pensar criticamente, para discernir entre la información más fehaciente, para aplicarla en situaciones prácticas y para redactar un texto académico basado en evidencias científicas aplicando las normas APA. Esta percepción, ha sido, sin duda, la base de la buena valoración del módulo. Como el propio alumnado menciona en sus valoraciones, es gratamente satisfactorio sentirse con mayor preparación y capacitación para enfrentarse a su futura labor docente. Los trabajos con una rigurosa planificación y coordinación docente, que diluyan las barreras entre disciplinas (Rodriguez et al., 2018) pueden ayudar a este fin y este TIM es muestra de ello. 


\section{Referencias}

American Psychological Association (2010). Manual de publicaciones. Washington: American Psychological Association.

Aristizabal, P., Rodríguez-Fernández, A. Rodríguez-Miñambres, P y Fernández-Zabala, A. (2015). El desarrollo de las competencias transversales en segundo curso de los Grados de Educación Infantil y Primaria. Revista Electrónica Interuniversitaria de Formación del Profesorado, 18 (3), 25-37.

Benito, Á., y Cruz, A. (2005). Nuevas claves para la docencia universitaria en el espacio europeo de educación superior: En el espacio europeo de educación superior (Vol. 10). Madrid: Narcea.

Booth, T. y Ainscow, M. (2002). Index for inclusion: Developing learning and participation in schools. Bristol: Centre for Studies on Inclusive Education (CSIE).

Chica Merino, E. (2011). Una propuesta de evaluación para el trabajo en grupo mediante rúbrica. EA, Escuela abierta: revista de Investigación Educativa, (14), 67-82.

Cowan, J. (2006). On becoming an innovative university teacher: Reflection in action: Reflection in action. Berkhire: Open University Press.

Dekker, S., Lee, N., Howard-Jones, P., y Jelle, J. (2012). Neuromyths in education: Prevalence and predictors of misconceptions among teachers. Frontiers in Psychology, 3. doi: 10.3389/fpsyg.2012. 00429

Delors, J. (1996). La educación encierra un tesoro. Paris: UNESCO.

Díaz Costa, E., Fernández Cano, A. Faouzi Nadim, T. y Caamaño Carillo, C. (2019). Modelamiento y estimación del índice de impacto de la investigación sobre la docencia. Revista Electrónica Interuniversitaria de Formación del Profesorado, 22(2), 211J228. doi: 10.6018/reifop.22.2.351671

EES (2019a). The Bolognia Declaration of June 19. Recuperado de: http://www.eees.es/pdf/bolognaEUA.pdf

EES (2019b). Estructuras Educativas Europeas. La respuesta de la universidad mediante el proyecto "Tuning". Recuperado de: http://www.eees.es/es/eees-estructuras-educativas-europeas

Eizagirre, A., Altuna, J., Pikabea, I., Marko, J. I., y Pérez, V. (2017). Las competencias transversales en el grado de Pedagogia: diagnóstico y estado de la cuestión. REDU. Revista de Docencia Universitaria, 15(1), 259-276.

Fernández, M. (2006). Desarrollo profesional docente. Granada: Grupo 
editorial universitario.

Fernández-Río, J., Cecchini, J. A., Méndez-Giménez, A., Méndez-Alonso, D. y Prieto, J. (2017). Diseño y Validación de un cuestionario de mediación del aprendizaje cooperativo en contextos educativos. Anales de Psicologia, 33(3), 680-688. doi: 10.6018/analesps

Flecha, R., Racionero, S., Tintoré, M., y Arbós, A. (2014). Actuaciones de Éxito en la Universidad. Hacia la Excelencia Tomando las Mejores Universidades como Modelo. Multidisciplinary Journal of Educational Research, 4(2), pp.131-150. doi: 10.4471/remie.2014.08

Fuentes, A., y Risso, A. (2015). Evaluación de conocimientos y actitudes sobre neuromitos en futuros/as maestros/as. Revista de Estudios e Investigación en Psicología y Educación, 6, 193-198. doi: 10.17979/reipe.2015.0.06.530

Gairín, J. (2011). Formación de profesores basada en competencias. Bordón. Revista de Pedagogía, 63(1), 93-108. Recuperado de:

https://core.ac.uk/download/pdf/78532495.pdf

González, J. y Wagenaar, R.(coord.) (2003). Tuning Educational Structures in Europe. Final Report Phase One. Bilbao: Universidad de Deusto.

Gobierno Vasco (2019). Plan Marco para el desarrollo de una escuela inclusiva. Vitoria-Gasteiz: Gobierno Vasco.

Howard-Jones, P. A. (2014). Neuroscience and education: myths and messages. Nature Reviews Neuroscience, 15(12), 817. doi: $10.1038 / \operatorname{nrn} 3817$

Johnson, D.W \& Johson, R.T. (2014): La evaluación en el aprendizaje cooperativo. España: Editorial SM.

Karakus, O., Howard-Jones, P. A., \& Jay, T. (2015). Primary and Secondary School Teachers' Knowledge and Misconceptions about the Brain in Turkey. Procedia-Social and Behavioral Sciences, 174, 1933- 1940. doi:.1016/j.sbspro.2015. 01.858

Morales, P. (2018). Aprendizaje basado en problemas (ABP) y habilidades de pensamiento crítico ¿una relación vinculante? Revista Electrónica Interuniversitaria de Formación del Profesorado, 21(2), 91-108. doi: 10.6018/reifop.21.2.323371

Moreno, T. (2012). La evaluación de competencias en educación. Sinéctica, Revista Electrónica de Educación, 39, 01-20. Recuperado de: http:/ /www.scielo.org.mx/scielo.php?pid=S1665109X2012000200010\&script=sci_arttext\&tlng=pt

Perines, H. (2017). Las murallas invisibles entre la investigación educativas y los docentes. Ciencia y Educación, 1(1), 11-21. 
El trabajo interdisciplinar de módulo como herramienta para el desarrollo de competencias transversales

Rekalde, I., Martínez, B., y Marko, J. I. (2012). Los proyectos interdisciplinares de módulo: Una experiencia innovadora en el Grado de Educación Social de la UPV/EHU. REDU: Revista de Docencia Universitaria, 10(3), 209-237.

Rodríguez, G., Ibarra, M. S., y Cubero, J. (2018). Competencias básicas relacionadas con la evaluación. Un estudio sobre la percepción de los estudiantes universitarios. Educación XXI, 21(1), 181-208. doi: 10.5944/educXX1.14457

Rodríguez-Miñambres, P., Rico-Martínez, A., López de Sosoaga-López de Robles, A. y Ugalde-Gorostiza, A.I. (2018). Cómo evaluar una simulación de la metodología por proyectos a través del uso de rúbricas en el Grado de Educación Primaria. Revista Electrónica Interuniversitaria de Formación del Profesorado, 21(2), 43-63. doi: 10.6018/reifop.21.2.315011

Ortega, M. D. (2010). Competencias emergentes del docente ante las demandas del espacio europeo de educación superior. Revista Española de Educación Comparada, 16, 305-328.

UNESCO (1990): Declaración Mundial sobre educación para todos. New York: UNESCO.

UNESCO (1994): Declaración de Salamanca y Marco de Acción para las necesidades educativas especiales. Paris: UNESCO.

UNESCO (2015). La educación para todos 2000-2015: Logros y desafios. Paris: UNESCO.

UNESCO (2016). Educación 2030. Declaración de Incheon y Marco de Acción para la realización del Objetivo de Desarrollo Sostenible 4. Garantizar una educación inclusiva $y$ equitativa de calidad y promover oportunidades de aprendizaje permanente para todos. Recuperado de: https://unesdoc.unesco.org/ark:/48223/pf0000245656_spa

UPV/EHU (2015). Informe de autoevaluación para la acreditación de Grado. Recuperado de: https://www.ehu.eus/es/grado-educacion-primariagipuzkoa/verificacion-seguimiento-y-acreditacion

UPV/EHU (2018). Plan estratégico de actuación. Recuperado de: https://www.ehu.eus/es/web/idazkaritza-nagusia/plan-estrategikoa2018-202 
